\title{
Trends in Alcoholic Hepatitis related Hospitalizations, Financial Burden, and Mortality in the United States
}

\author{
Raxitkumar Jinjuvadia, MD, MPH ${ }^{1}$ and Suthat Liangpunsakul, MD, MPH ${ }^{2,3}$ \\ ${ }^{1}$ Division of Gastroenterology and Hepatology, Henry Ford Hospital, Detroit, Michigan \\ ${ }^{2}$ Division of Gastroenterology and Hepatology, Department of Medicine, Indiana University \\ Medical Center, Indianapolis, Indiana \\ ${ }^{3}$ Roudebush Veterans Administration Medical Center, Indianapolis, Indiana
}

\begin{abstract}
Background-Alcoholic hepatitis (AH) is the most florid manifestation of alcoholic liver disease which accounts for significant morbidity, mortality and financial burden. Aim of this study is to evaluate temporal trend of hospitalizations from alcoholic hepatitis and evaluate its financial impact.
\end{abstract}

\begin{abstract}
Methods-The National Inpatient Sample (NIS) databases (from 2002 to 2010) which are collected as part of Healthcare Cost and Utilization Project by Agency for Healthcare Research and Quality were utilized. Individuals with age $\geq 21$ years were included. The hospitalizations with primary diagnosis of AH were captured by ICD-9 codes. The national estimates of hospitalization were derived using sample weights provided by NIS. Simple linear regression method was used to assess trends in mortality and length of stay over time.
\end{abstract}

Results-We observed the increased in total cases of AH-related hospitalization from 249,884 ( $0.66 \%$ of total admission in 2002) to 326,403 ( $0.83 \%$ of total admission in 2010). The significant increase in the total admission rate was attributable mainly to the rise in inpatient hospitalization for secondary diagnosis of $\mathrm{AH}(0.48 \%$ in 2002 to $0.67 \%$ in 2010). Most of the AH related hospitalization were males. Hepatic encephalopathy was found to be the most common admitting diagnosis for individuals hospitalized with secondary diagnosis of $\mathrm{AH}(8.9 \%$ in 2002 and $8.6 \%$ in 2010). There was a significant decrease in inpatient mortality for primary diagnosis of AH from $10.07 \%$ (in 2002) to $5.76 \%$ (in 2010) (absolute risk reduction: 4.3\%). Average cost of hospitalization related to primary diagnosis of $\mathrm{AH}$ was \$27,124 and \$46,264 in 2002 and 2010, respectively. After adjusting for inflation, the additional cost of each hospitalization seemed to increase by $40.7 \%$ in 2010 compared to 2002 (additional cost per hospitalization $\$ 11,044$ in 2010

Corresponding Author: Suthat Liangpunsakul, MD MPH, Division of Gastroenterology and Hepatology, 550 N. University Blvd, UH 4100, Indianapolis, IN 46202, Phone: (317) 278-1630, Fax: (317) 988-3180, sliangpu @iupui.edu.

Author contribution:

$\mathrm{RJ}$ and SL - study design

RJ and SL - literature search, review and collection of data

RJ and SL - analysis and interpretation of data

$\mathrm{RJ}$ and SL - drafted manuscript

$\mathrm{SL}$ - made critical revisions to manuscript

Conflict of interest: none 
compared to 2002). Federal (Medicare) or state (Medicaid) supported health insurance program are the main primary expected payers for these AH hospitalizations $(\sim 25 \%-29 \%)$. Despite increase in cost per hospitalization, length of stay for hospitalization due to primary diagnosis of AH was not observed to decrease substantially over time (6.7 days in 2002 to 6.1 days in 2010).

Conclusion-AH-related hospitalization continued to increase during the study period, despite the decreasing in the in-hospital mortality rate. Substantial increases in healthcare cost and utilization among hospitalized AH patients were observed.

\section{Keywords}

alcoholic hepatitis; financial burden; National Inpatient Sample database

\section{BACKGROUND}

Alcoholic liver disease includes a spectrum of liver disease ranging from reversible fatty liver to alcoholic hepatitis (AH), and cirrhosis ${ }^{1}$. AH presents as an acute hepatic injury in patients who consume excessive amounts of alcohol ${ }^{2}$. In mild cases, patients have a favorable outcome with alcohol abstinence. However, those with severe disease have a high 30-day mortality rate ${ }^{1}$.

There are no strong epidemiological data on the incidence of AH in the US. In Denmark, the annual incidence rate of alcoholic hepatitis rose from 37 to 46 per 100,000 for men and from 24 to 34 per 100,000 for women, during the study period from 1999-2008 ${ }^{3}$. Further, the overall 5-year mortality was 56\% (47\% in those without cirrhosis, and $69 \%$ in cases with (irrhosis) ${ }^{3}$.

While the healthcare and economic burden for other liver diseases such as hepatitis B or C are expected to decrease in the next decade ${ }^{4}$, the problem of liver disease attributable to the use of alcohol will remain significant ${ }^{5}$. Recent studies demonstrated the increasing levels of alcohol consumption in the United States ${ }^{6,7}$. More importantly, drinking is starting at an earlier age ${ }^{8-10}$ with binging becoming more common pattern ${ }^{11}$. Taken together, these trends in alcohol consumption will likely cause substantial health, social, and economic burdens related to alcoholic liver disease, with increasing numbers of patients requiring hospitalization and the need for the outpatient care to provide support for these patients ${ }^{5}$.

We previously examined the clinical characteristics and risk factors associated with mortality in hospitalized alcoholic hepatitis patients in the United States using the 2007 National Inpatient Sample ${ }^{12}$. In that study, we found that hospitalized AH patients resulted in significant healthcare cost and utilization ${ }^{12}$. The average total charges during hospitalization for $\mathrm{AH}$ were higher than that from acute myocardial infarction, acute cerebrovascular disease, and acute pancreatitis ${ }^{13}$.

Because of the current limitations and therapeutic options for patients with $\mathrm{AH}$, the overall mortality especially in those with severe AH remains high ${ }^{1}$. However, the data regarding inpatient mortality, healthcare utilization for patients with $\mathrm{AH}$ over the past decade are lacking. We hypothesized that i) the rate of inpatient admission secondary to $\mathrm{AH}$ increased over time and ii) the in-hospital mortality secondary to AH should not change due to the 
limitation in the effectiveness of the currently available therapies. We analyzed the NIS data from 2002-2010 to determine the trends in rate of inpatient admission, outcome, hospitalization costs, financial burden, and mortality for hospitalized $\mathrm{AH}$ patients in the United States.

\section{METHODS}

\section{Data source}

The NIS database is part of the Healthcare Cost and Utilization Project (HCUP), sponsored by the Agency for Healthcare Research and Quality (AHRQ) ${ }^{14}$. The Nationwide Inpatient Sample (NIS) is the largest all-payer inpatient care database in the United States, containing data on more than seven million hospital stays annually from approximately 1,000 hospitals, constituting a $20 \%$ stratified sample of all U.S. hospitals. All data were weighted using discharge level values, based on the relative proportion of the total U.S. hospital patient population accounted for by that record, to produce $100 \%$ national estimates.

Subjects who were 21 years old or older who were hospitalized with the diagnosis of $\mathrm{AH}$ from 2002-2010 were included. AH was based on the following International Classification of Diseases-Ninth Revision, Clinical Modification (ICD-9-CM) discharge diagnosis codes (571.1). If this code was listed in the first position in the database, the admission was considered to be a primary AH hospitalization; otherwise, the admission was considered to be a secondary $\mathrm{AH}$.

One of the outcomes in our study was the inpatient mortality in subjects with AH. The following ICD-9 codes were also used to identify known risk factors related to mortality in these patients: acute renal failure (584.50-70, 584.80-90, 586.00, and 593.90), GI bleeding (578.0-9), and sepsis (38.00-38.90).

Patient age, race (white, black, Hispanic, others), gender, household income, geographic region of treatment (Northeast, South, Midwest, and West) were abstracted. The types of hospitals were categorized into teaching/community and urban/rural location. The primary payer for the hospitalization was categorized as Medicare, Medicaid, private insurance, selfpay, or other. Outcome-related measures were presented separately for both primary and secondary diagnoses of AH which included in-hospital mortality, length of stay, and discharge disposition. Discharge disposition was categorized as routine, short term hospital, skilled nursing facility, and home health care.

\section{Statistical analysis}

All statistical analyses were performed using the sampling weights and stratified sample design of the NIS to obtain nationally representative estimates. Descriptive statistics were presented as mean $\pm \mathrm{SD}$ for continuous variables and frequencies for categorical variables. Annual rates of primary and secondary AH hospitalizations were calculated by dividing the number of hospitalizations with AH by the total inpatient admissions in the US in a given year. Changes in hospitalization rates and healthcare costs between 2002 and 2010 were determined with simple regression analysis. Statistical analyses were performed using SAS 9.3 (Cary, NC). 


\section{RESULTS}

\section{National estimates of AH-related hospitalization rates in the US from 2002-2010}

We observed the increased in total cases of AH-related hospitalization from 249,884 (0.66\% of total admission in 2002) to 326,403 ( $0.83 \%$ of total admission in 2010). The significant increase in the total admission rate was attributable mainly to the rise in inpatient hospitalization for secondary diagnosis of $\mathrm{AH}(0.48 \%$ in 2002 to $0.67 \%$ in 2010 , Table 1$)$. Hepatic encephalopathy was found to be the most common admitting diagnosis for individuals hospitalized with secondary diagnosis of AH (8.9\% in 2002 and 8.6\% in 2010). We observed no changes in the rate of admission for primary diagnosis of $\mathrm{AH}$ during this period $(\sim 0.16-0.18 \%)$.

\section{Demographics and clinical characteristics of hospitalized AH patients in the US}

The mean age of patients hospitalized with a diagnosis of AH over this period was 53 years old. The majority of hospitalized patients were men $(73 \%)$ and white $(65 \%-70 \%)$. The majority of cases had the household income in the first ( 32\%) quartile (Table 2).

\section{Sources of admission and types of facilities for hospitalized AH patients}

Hospitalized AH patients were admitted through local emergency department (64\%-74\%). There were no significant changes in the source of admission over this period except that the admission through emergency room was significantly decreased in 2010. Majority of cases were hospitalized into the hospitals which were located in the urban area (86\%-90\%) (Table $3)$.

Fifty to sixty percent of cases were admitted into 'non-teaching' hospitals. There were significant geographic variations in hospitalized cases. We found that $33 \%-38 \%$ of hospitalized cases were in the Southern states; which was significantly higher than that admitted to other regions ( $\mathrm{p}<0.05)$ (Table 3).

\section{Healthcare costs and outcomes related to hospitalizations from AH in the US}

During the study period, there were no changes in the average length of stay for overall AH admissions (6.6 days in 2002 and 5.9 days in 2010). There were no differences in the length of hospital stay among patients with either primary or secondary diagnosis of AH (Table 4).

Majority of patients $(62 \%-65 \%)$ were discharged to home with outpatient follow up visit. About $13 \%-14 \%$ of these cases were transferred to skilled nursing facility upon discharge. Though most patients had medicare; there were no differences in the payer sources among medicare carriers and those with medicade or private insurers $(\mathrm{p}<0.05$, Table 4$)$.

The overall in-hospital mortality of AH cases was significantly decreased from $8 \%$ in 2002 to $5.1 \%$ in 2010 ( $\mathrm{p}<0.05)$. In the detailed analysis, in-hospital mortality rates significantly decreased for both primary $(10.1 \%$ in 2002 to $5.8 \%$ in 2010, p < 0.05$)$ and secondary AH ( $7.2 \%$ in 2002 to $4.9 \%$ in 2010, p < 0.05) hospitalizations (Table 4). Among patients who died during the hospitalization, the rate of renal failure was noted to decrease significantly 
from $43.6 \%$ in 2002 to $34.3 \%$ in 2010 . However, the rate of sepsis among these cases increased from $26.7 \%$ in 2002 to $37.8 \%$ in 2010 (Table 5, Figure 2).

Despite no substantial changes in the length of stay, the overall hospitalization cost was increased substantially from $\$ 25,276$ in 2002 to $\$ 40,870$ in 2010 . The total hospital charges for patients with primary diagnosis of $\mathrm{AH}(\$ 27,124-\$ 46,264)$ were significantly higher than those with secondary diagnosis $(\$ 24,594-\$ 39,539)$ of AH during the study period ( $\mathrm{p}<$ 0.05, Table 4).

\section{DISCUSSION}

AH continues to be a cause of considerable mortality and morbidity especially in heavy drinkers ${ }^{1}$. Our study demonstrated an increasing trend in AH-related hospitalizations in the US from 2002-2010, primarily due to secondary diagnosis of AH. We observed that the inpatient mortality rate was significantly decreased during this period. However, financial charges among hospitalized AH subjects remained high and increased substantially, despite little changes in the total length of stay.

The increase in hospitalization rate of $\mathrm{AH}$ over the past decade attests the notion that $\mathrm{AH}$ continues to be a major health problem in the US. This observation coincides with the report on the increasing levels of alcohol consumption ${ }^{7}$ and also binge drinking ${ }^{11}$ in the US. Though recent data showed the shift in alcohol drinking starting at the younger age, we found no difference in the mean age of hospitalized AH patients during the study period. In general, women are more susceptible than men to the adverse effects of alcohol and they develop AH after a shorter period and smaller amounts of alcohol use than men. However, in our study, we observed significantly higher proportion of male in hospitalized $\mathrm{AH}$ patients. This finding is not surprising as it has been shown that more men drink alcohol above the 'safety range' than women ${ }^{15}$.

In agreement with our previous report, hospitalized $\mathrm{AH}$ patients result in significant healthcare cost and utilization ${ }^{12}$. Patients with AH were hospitalized in different geographic locations especially in the urban areas. We found that federal (Medicare) or state (Medicaid) supported health insurance program are the main primary expected payers. Our findings were compatible with the levels of socioeconomic status which was measured by household income national quartile of the study cohort; in which majority of patients had the annual household income in the first quartile range.

During the study period, we found that financial charges among hospitalized AH subjects remained high, despite little changes in the total length of stay. It is likely that the rising in hospital charges is secondary to the rising in healthcare cost in the United States over the past decade ${ }^{16}$.

It is surprising to observe the decrease in overall mortality among hospitalized AH patients over the past decade, despite the limitation or the new advancement in the treatment of $\mathrm{AH}^{1}$. Using the NIS dataset, we have previously shown that the significant increase in mortality of AH patients who developed infections (i.e. sepsis or SBP) and acute renal failure during the hospital stay ${ }^{12}$. Our observations regarding the decreasing in mortality 
might be contributed to the early treatment of infections or fluid management to prevent the onset of acute renal failure. To support this hypothesis, we analyzed several known factors which are associated with mortality among patients with $\mathrm{AH}$. Interestingly, we observed the decreasing trends of acute renal failure among cases who died during the hospital stay from 2002-2010. However, our results need to be cautiously interpreted. First, we do not have access to the etiology of death of these patients in the NIS database. The presence of acute renal failure and/or sepsis during the hospital stay might, in fact, not relate to the actual cause of death since we do not have the information whether renal failure/sepsis has resolved during the hospitalization. Second, NIS data only captured clinical presentations without any laboratory results for each patient. Therefore, we might not be able to perform the severity- adjusted (such as using the MELD scores) in-hospital mortality.

This study has a few potential limitations. Our analysis may be limited by the accuracy of the NIS database, which is based primarily on medical record coding. We acknowledge the deficiencies of using ICD-9 codes to identify secondary diagnosis of AH during the hospitalization. In certain circumstances, the ICD-9 for jaundice and hepatic encephalopathy were used as the primary admission diagnosis, and in fact such presentation might be secondary to underlying AH. One would argue that the overall mortality rate for subjects with $\mathrm{AH}$ in our study was much lower than previously reported ${ }^{1,2}$. We would like to emphasize that the mortality rate based on the NIS data was only confined to those cases who died while hospitalization. In general, the short term (30-day mortality) for severe alcoholic hepatitis is $\sim 30 \%-50 \% 1,15$. Unfortunately, the NIS dataset did not provide any information regarding patient's outcomes once he/she was released from the hospital. Despite these shortcomings, our study is strengthened by the use of a database that represents a wide variety of U.S. medical centers and patient populations.

In summary, we found the increase in AH-related hospitalization during the study period, despite the decreasing in the in-hospital mortality rate. Our results documented significant healthcare cost and utilization among hospitalized AH patients. Our findings should alert healthcare provider for better screening, education, and interventions among abusive drinkers aimed at promoting alcohol abstinence, thus preventing episodes of $\mathrm{AH}^{15}$.

\section{Acknowledgments}

Grant Support: This study is supported by K08 AA016570 from the NIH/NIAAA, 1I01CX000361-01 from the Veterans Affairs Research and Administration, and W81XWH-12-1-0497 from United States Department of Defense (All to S.L).

\section{References}

1. Singal AK, Kamath PS, Gores GJ, Shah VH. Alcoholic hepatitis: current challenges and future directions. Clin Gastroenterol Hepatol. 2014; 12(4):555-564. [PubMed: 23811249]

2. Gao B, Bataller R. Alcoholic liver disease: pathogenesis and new therapeutic targets. Gastroenterology. 2011; 141(5):1572-1585. [PubMed: 21920463]

3. Sandahl TD, Jepsen P, Thomsen KL, Vilstrup H. Incidence and mortality of alcoholic hepatitis in Denmark 1999-2008: a nationwide population based cohort study. J Hepatol. 2011; 54(4):760-764. [PubMed: 21126790]

4. Neff GW, Duncan CW, Schiff ER. The current economic burden of cirrhosis. Gastroenterol Hepatol (N Y ). 2011; 7(10):661-671. [PubMed: 22298959] 
5. Williams R. The pervading influence of alcoholic liver disease in hepatology. Alcohol Alcohol. 2008; 43(4):393-397. [PubMed: 18385413]

6. Xuan Z, Nelson TF, Heeren T, et al. Tax policy, adult binge drinking, and youth alcohol consumption in the United States. Alcohol Clin Exp Res. 2013; 37(10):1713-1719. [PubMed: 23711219]

7. Yoruk BK. Legalization of Sunday alcohol sales and alcohol consumption in the United States. Addiction. 2014; 109(1):55-61. [PubMed: 24103041]

8. Siegel M, DeJong W, Naimi TS, et al. Alcohol brand preferences of underage youth: results from a pilot survey among a national sample. Subst Abus. 2011; 32(4):191-201. [PubMed: 22014249]

9. Siegel MB, Naimi TS, Cremeens JL, Nelson DE. Alcoholic beverage preferences and associated drinking patterns and risk behaviors among high school youth. Am J Prev Med. 2011; 40(4):419426. [PubMed: 21406275]

10. Miller TR, Levy DT, Spicer RS, Taylor DM. Societal costs of underage drinking. J Stud Alcohol. 2006; 67(4):519-528. [PubMed: 16736071]

11. Bor J, Basu S, Coutts A, McKee M, Stuckler D. Alcohol use during the great recession of 20082009. Alcohol Alcohol. 2013; 48(3):343-348. [PubMed: 23360873]

12. Liangpunsakul S. Clinical characteristics and mortality of hospitalized alcoholic hepatitis patients in the United States. J Clin Gastroenterol. 2011; 45(8):714-719. [PubMed: 21085006]

13. Fagenholz PJ, Fernandez-del CC, Harris NS, Pelletier AJ, Camargo CA Jr. Direct medical costs of acute pancreatitis hospitalizations in the United States. Pancreas. 2007; 35(4):302-307. [PubMed: 18090234]

14. Healthcare Cost and Utilization Project (HCUP) databases. Agency for Healthcare Research and Quality; Available at: http://www.hcup-us.ahrq.gov/nisoverview.jsp

15. Lucey MR, Mathurin P, Morgan TR. Alcoholic hepatitis. N Engl J Med. 2009; 360(26):27582769. [PubMed: 19553649]

16. Kumar S, Ghildayal NS, Shah RN. Examining quality and efficiency of the U.S. healthcare system. Int J Health Care Qual Assur. 2011; 24(5):366-388. [PubMed: 21916090] 

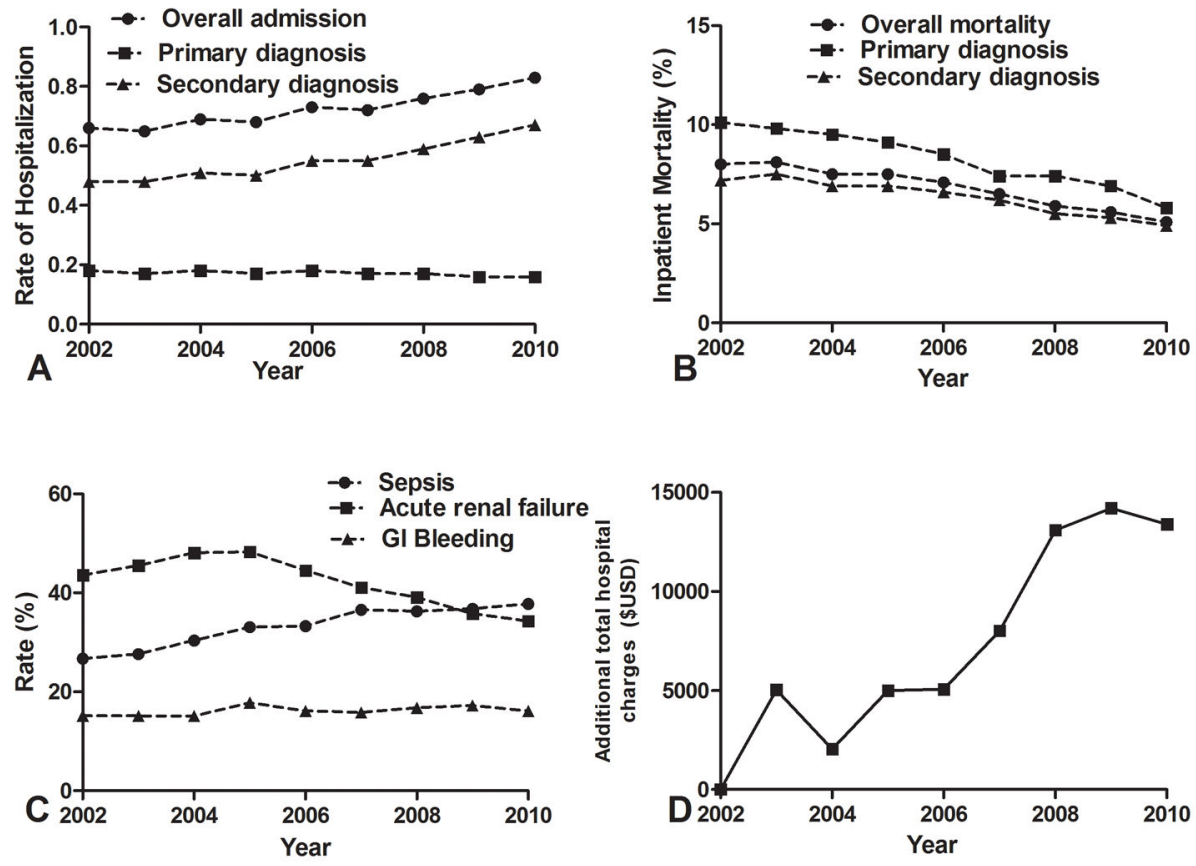

Figure 1.

(A) Rate of hospitalization for the diagnosis of AH in the United States from 2002-2010, (B) Inpatient mortality for the diagnosis of AH from 2002-2010, (C) Rate of sepsis, renal failure and GI bleed among patients who died during hospitalization from 2002-2010, and (D) Additional total hospital charges for the diagnosis of AH from 2002-2010, using the charges in 2002 as the reference. 


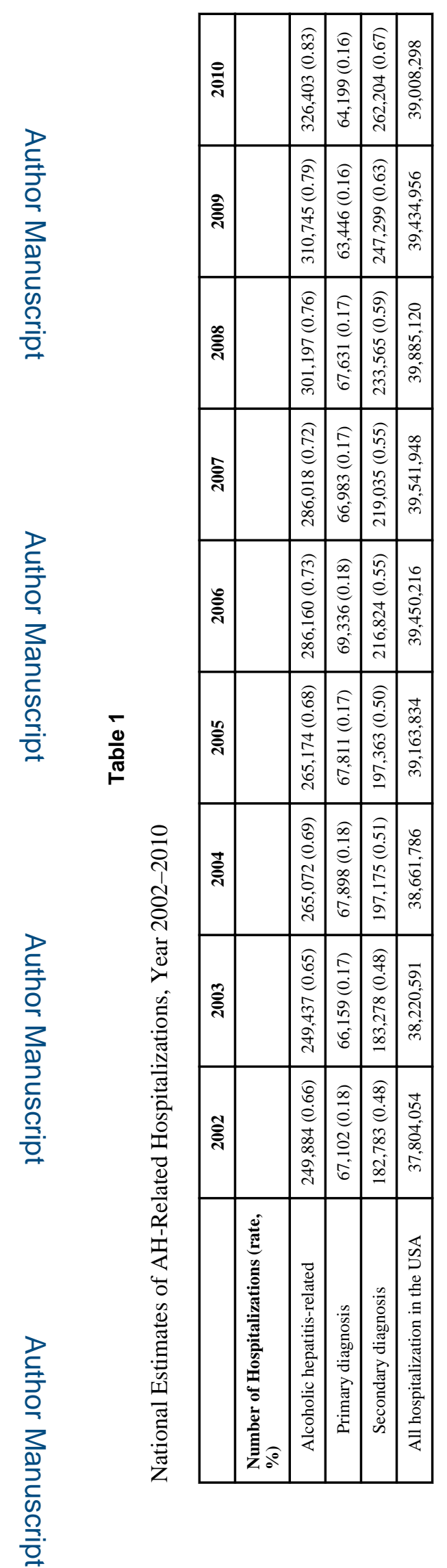

J Clin Gastroenterol. Author manuscript; available in PMC 2016 July 01. 
ฮิ 


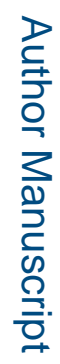

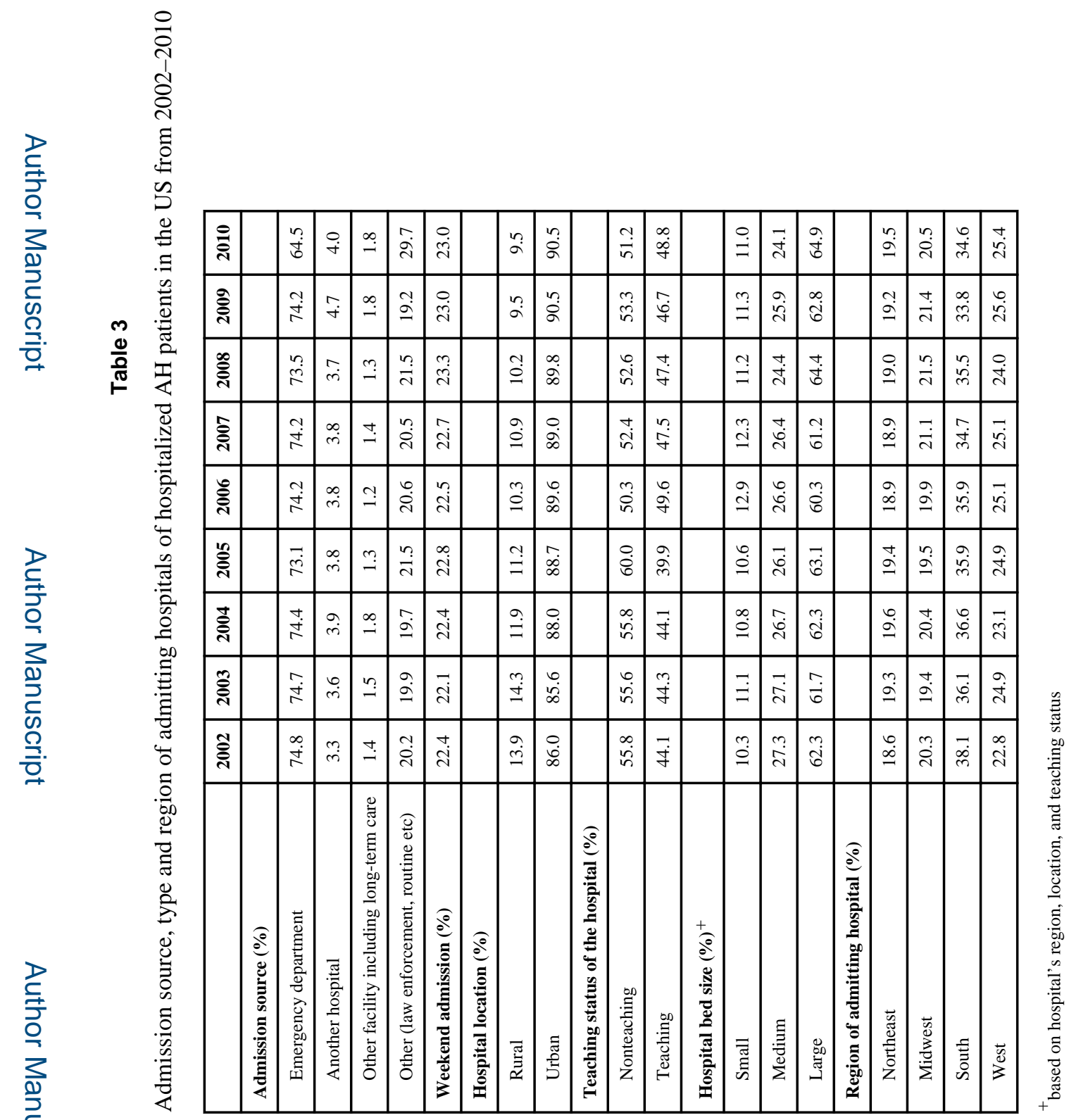

J Clin Gastroenterol. Author manuscript; available in PMC 2016 July 01. 


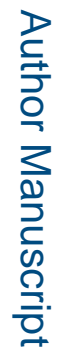

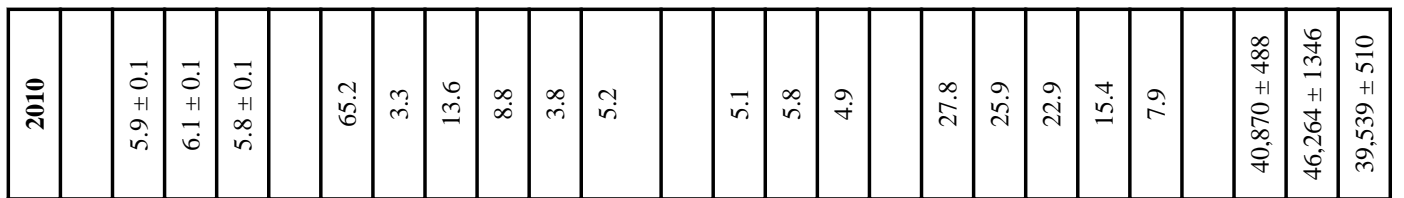

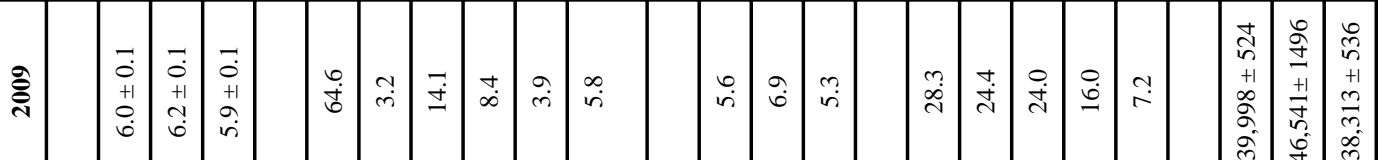

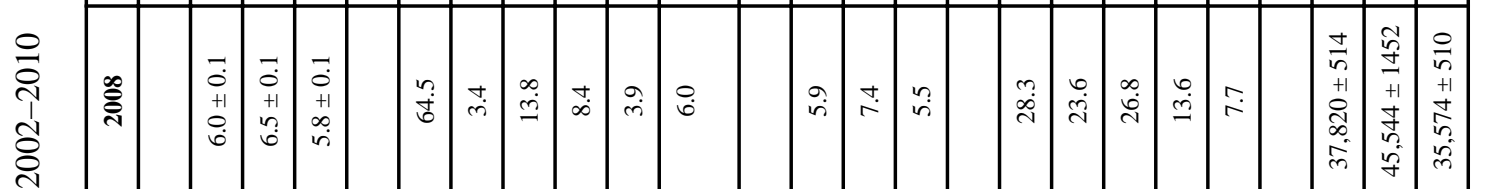

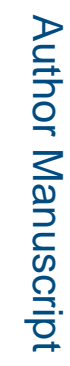

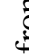

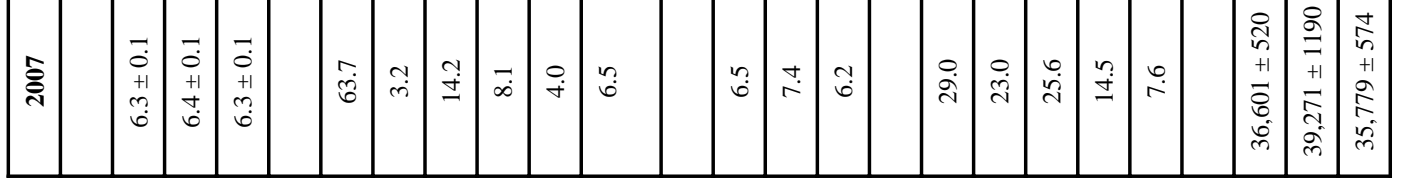

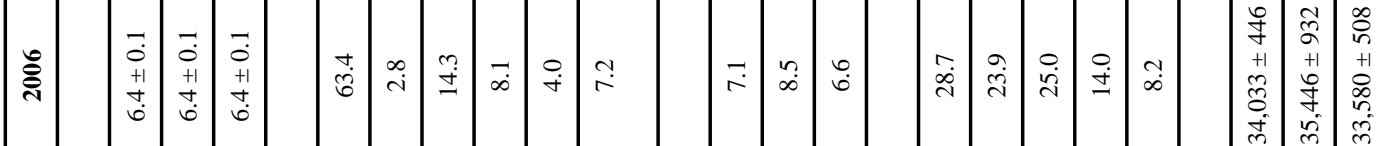

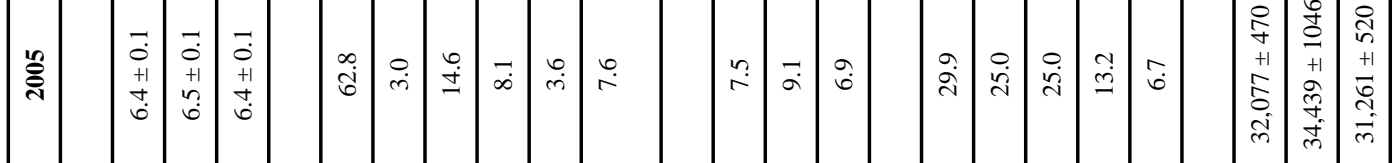

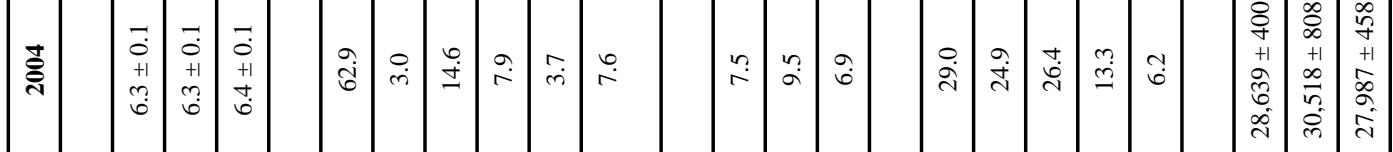

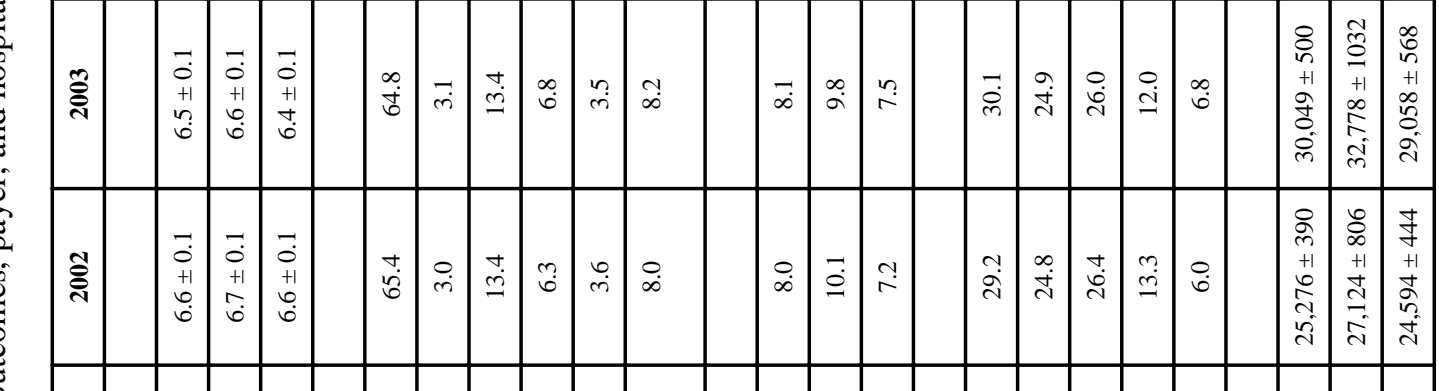

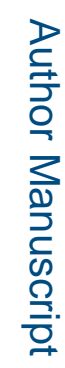

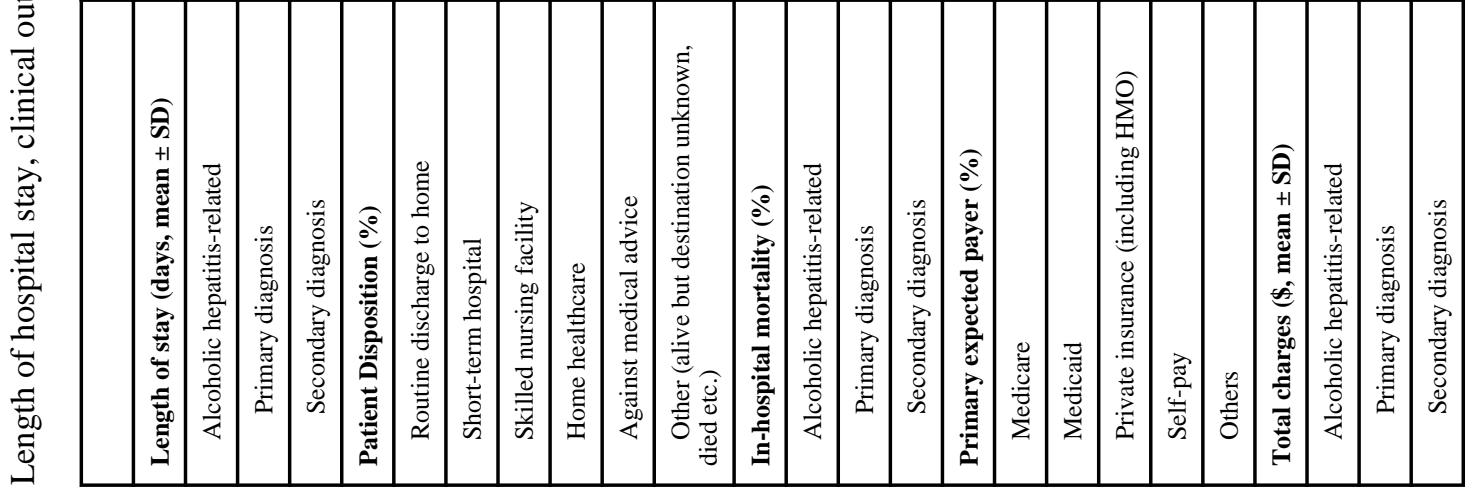

J Clin Gastroenterol. Author manuscript; available in PMC 2016 July 01. 

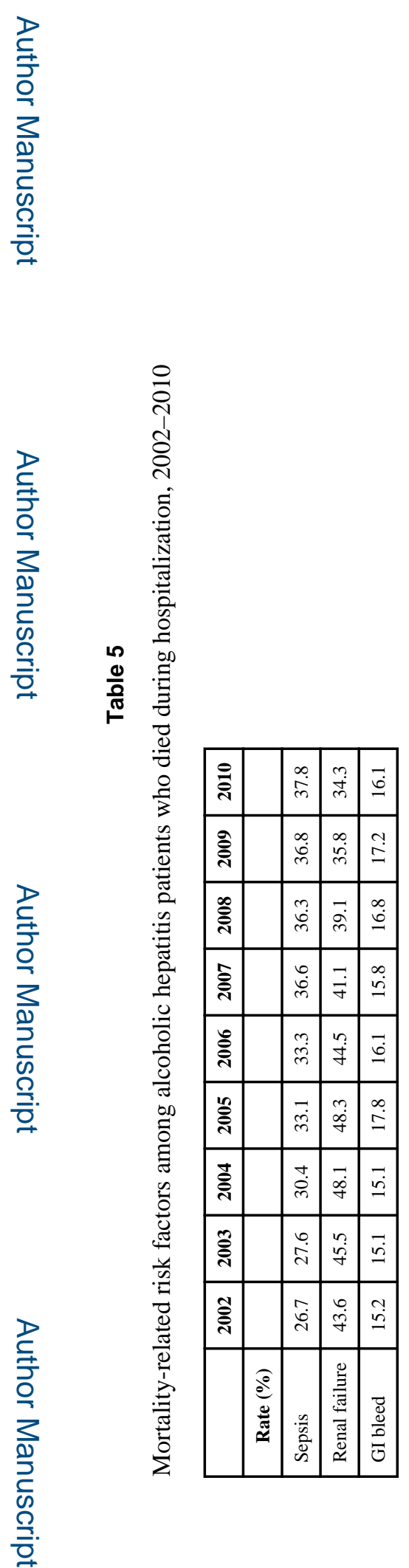

J Clin Gastroenterol. Author manuscript; available in PMC 2016 July 01. 